(c) 2012 IEEE. Personal use of this material is permitted. Permission from IEEE must be obtained for all other uses, in any current or future media, including reprinting/republishing this material for advertising or promotional purposes, creating new collective works, for resale or redistribution to servers or lists, or reuse of any copyrighted component of this work in other works. 


\title{
Experimental performance of compact UWB antenna for Breast Cancer screening
}

\author{
S. K. Padhi ${ }^{1}$, Atsushi Mase ${ }^{2}$, N. Ito ${ }^{2}$ and Dan Zhang ${ }^{2}$
}

\begin{abstract}
This paper presents experimental performances of the algorithm used for detection of malignant tissue in breast. The algorithm uses ultra-short pulse radar (USPR) technique along with confocal microwave imaging (CMI) algorithm based on synthetic aperture radar (SAR) to detect tumor cells or tissue. A compact Vivaldi antenna having ultra-wideband (UWB) performance with bandwidth $3 \mathrm{GHz}$ to $8 \mathrm{GHz}$ is used as transceiver and an impulse with no distortion is used as an excitation signal in our experiment. We used various synthetic breast phantom and metallic or high-k dielectric balls as tumor. The skin subtraction method using Teflon cover seems to be promising for this experiment.
\end{abstract}

\section{INTRODUCTION:}

Microwave imaging of the complex permittivity profile of an unknown object buried under dielectric interface or half-space is an important technology that has been used successfully in subsurface sensing such as GPR (Ground Penetrating Radar) used in mine detection [1]. Other potential applications include nondestructive testing of materials, biomedical imaging, [2] and the detection of defects and cracks in construction materials [3]. Recently, the approach has received considerable attention due to its potential advantages over more established techniques, such as $\mathrm{X}$-ray tomography, as an alternative tool in medical diagnostics such as the detection of breast cancers. Today breast cancer is the most common form of cancer among women in most of countries, and the standard method of diagnosing breast cancer, is by Xray mammography [4]. Despite its wide-scale usage, there are limitations in this technique; for example, (a): upto $15 \%$ cases the tumors cannot be diagnosed properly due to false-negatives cases such as, low contrast between the malignant and healthy tissue, or with false positive cases, where the suspected tumors are found to be benign in further examinations [4]; (b): difficulty in imaging for women with dense breast and inconclusive results and etc. Therefore from a diagnostic point of view, these limitations provide clear motivations for investigation for alternate or complimentary tools for imaging.
In general, two different approaches are used to detect the dielectric contrast between the object and the background medium: (i) radar-like backscattering methods and (ii) tomographic methods. In the radarlike methods, an ultra-wideband signal illuminates the object from several directions and the amplitude and the arrival time of the reflected signals are used to identify the location of the strong scatterer [5]. In the tomographic approach, the measured scattering data is used to recover quantitative images of the dielectric profile using either linear or non-linear approximations. Linear techniques such as Born and Rytov approximations have been used widely in the image reconstruction process for weak scatterers as first order approximation [6]. However, for large objects with high contrast ratio, such as often found in biological tissue structures, iterative nonlinear methods are used which usually involve the minimization of cost function [6-7]. This paper primarily focuses on radar type backscattering method for breast imaging.

An ultrashort-pulse radar (USPR) having frequencies in microwave region is a promising method for non-destructive detection with imaging diagnostics, due to good spatial resolutions [8-9]. The shorter the incident pulse-width is, the wider the band extends, thereby producing greater information as well as better resolution about the reflected objects. We use an impulse with picosecond (ps) pulse width as a source and measure the reflected waves from the discontinuous layer of different dielectric constant. In addition, the impulse contains little power energy due to its so small duty cycle. Therefore, it is appropriate for imaging diagnostics of living human body in order to detect abnormal tissue, such as, breast cancer.

In this paper, the numerical simulation and the experiments of breast cancer detection using the USPR with a compact Vivaldi antenna having ultra-wideband (UWB) performance are described. The antenna has a wide bandwidth with frequency band from $3 \mathrm{GHz}$ to 8 GHz. An impulse with no distortion is used as an incident wave for two-dimensional simulation study to perform the image reconstruction of the breast. In our experiments, we use various synthetic breast phantoms

\footnotetext{
${ }^{1}$ CIRA/ICRAR, Curtin University, Perth, Australia Email: shantanu_padhi@ieee.org.

${ }^{2}$ Art Science and Technology Center for Cooperative Research (KASTEC), Kyushu University, Fukuoka, Japan Email: mase@astec.kyushu-u.ac.jp.
} 
including laboratory made and commercially available phantoms, and metallic or high-k dielectric balls as tumor. The image reconstruction algorithm is based on confocal microwave imaging (CMI) algorithm as used in synthetic aperture radar (SAR) [11-14].

\section{MEASUREMENT SETUP:}

The procedures involve the collections of backscattered signal after illuminating the object with an ultra-wideband short pulse.

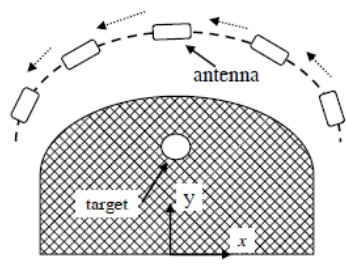

(a)

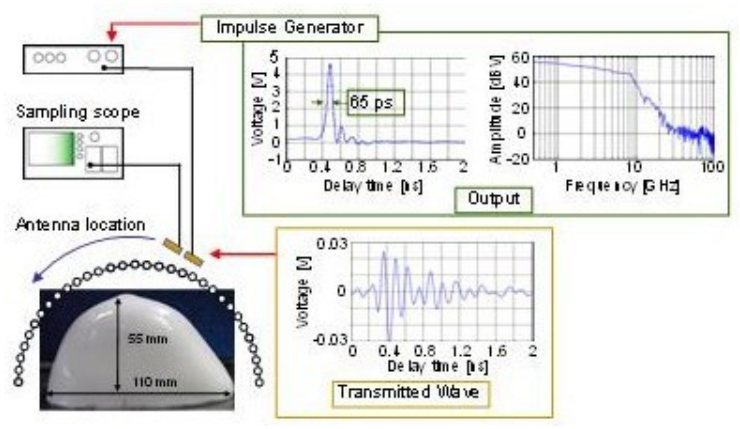

(c)

Fig. 1. The simulation setup; (a): the breast phantom with tumor and location of antennas, (b): breast phantom made by CRIS [15], (c): experimental setup used for measurements.

First, the relative distances between the focal point and each antenna position are determined and then converted to the time delays. The reflected waves obtained from each antenna are summed up coherently to determine the pixel value at each focal point. The reconstruction algorithm used in this study is based on confocal microwave imaging (CMI) algorithm as used in synthetic aperture radar (SAR) [11-13]. The intensity $S$ of the pixel is given in eq(1).

$$
\begin{aligned}
& S \diamond={ }_{m=1}^{M} \diamond_{m}\left(\diamond_{m} \diamond\right)^{2} \\
& \diamond_{m} \diamond=\frac{-\diamond_{m}}{\Delta \diamond}
\end{aligned}
$$

Where, $s_{m}$ is back scattered waveform at the $\mathrm{m}^{\text {th }}$ antenna located at $r_{m} ; t_{m}(r)$ is the time delay from $m^{\text {th }}$ antenna to the focal point of interest (r) and $\Delta t$ is the sampling time. For excitation, we use Gaussian ultrawideband pulse as shown in equation 3 .

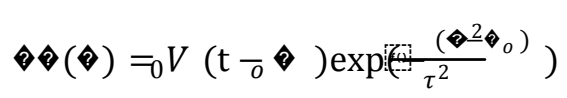

where, Vo is used to adjust the amplitude of pulse and $\tau$ is 65 ps. The geometry of the problem used in FDTD simulation is shown in Fig. 1(a). A semicircle shape with radius of $10 \mathrm{~cm}$ is used as a breast model in our FDTD code. The material property of normal breast tissue is assumed as $\varepsilon_{\mathrm{r}}=9, \sigma=0.4 \mathrm{~S} / \mathrm{m}$ and that of the malignant breast tissue is $\varepsilon_{\mathrm{r}}=50.0$, and $\sigma=$ $4 \mathrm{~S} / \mathrm{m}[3,6]$, where $\varepsilon_{\mathrm{r}}$ and $\sigma$ are dielectric permittivity and conductivity respectively for given tissue types. The full width half-maximum (FWHM) of pulse covers the frequency band of 3-8 GHz. The Tx antenna is rotated 5 degree increment around the breast from 0 to 180 degrees. The reflected scattered waves are collected at the $\mathrm{Rx}$ antenna port.

Once the reflected signals are collected, the post processing routines are used to extract the permittivity and conductivity profiles.

\section{NUMERICAL RESULTS AND DISCUSSION:}

For experimental testing, we used the commercially available phantom (CRIS model-051) [15] as shown in Fig 1(b). The phantom has relative dielectric constant close to 20 and attenuation of $19 \mathrm{db} / \mathrm{cm}$ at 8 GHz. The prototypes including antennas are immersed in matching liquid (cooking oil) with relative dielectric constant of 3.0 and attenuation of $0.05 \mathrm{~dB} / \mathrm{cm}$ at $8 \mathrm{GHz}$. The antennas are placed 20 $\mathrm{mm}$ above the outer skin of phantom. This distance will be sufficient to provide a reasonable coverage by the radiation pattern of transmit antenna.

A metallic ball with $9 \mathrm{~mm}$ in diameter is used as a tumor and is placed around $10 \mathrm{~mm}$ from the top of the breast phantom. Both transmitting (Tx) and receiving (Rx) antennas are attached to rotational axis of stepping motor. During the measurements both antennas are used to move with 5 degree step sequentially around the breast phantom. The reflected waves from the breast model are received at each antenna location. An $8 \mathrm{~V}$ magnitude of pulse with 65ps FWHM is used in our experiment. 
The Vivaldi antenna is used as both transmitter and receiver. The antenna was fabricated on $1.57 \mathrm{~mm}$ thick Taconic TLX substrate with dielectric constant of 2.7. The antenna uses a modified version of stub for broad band operation. The impedance characteristics of both $\mathrm{Rx}$ and $\mathrm{Tx}$ antennas are measured with Anritsu network analyzer and are shown in Fig. 2 along with maximum gain data. As can be seen, S-parameter performances of both antennas are in agreement with calculated performances. The frequency spectrums are in acceptable range for measurements. The antenna shows maximum gain of $9.6 \mathrm{dBi}$ and agrees well with simulated data. The scattered signals are detected by a high-speed sampling oscilloscope.

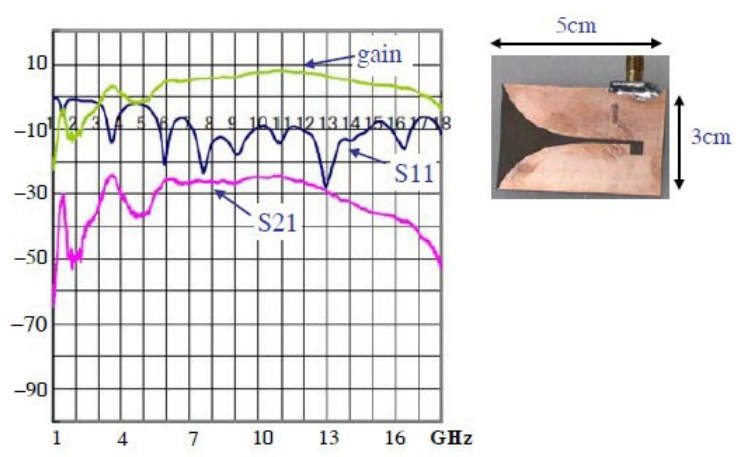

Fig. 2: Measured performance of Vivaldi antenna in both $\mathrm{Rx}$ and Tx configuration.

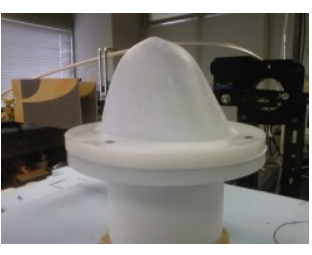

(a)

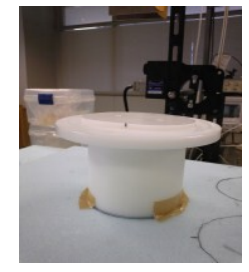

(b)
No Object, Use Teflon Cover
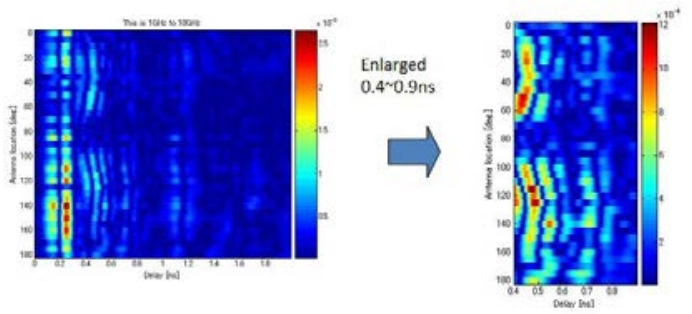

(c)

Fig. 3 (a-b): The measurement setup for Teflon cover used for background subtraction; (c): time delay for Teflon cover only.
Object placed at $5 \mathrm{~mm}$ from the surface

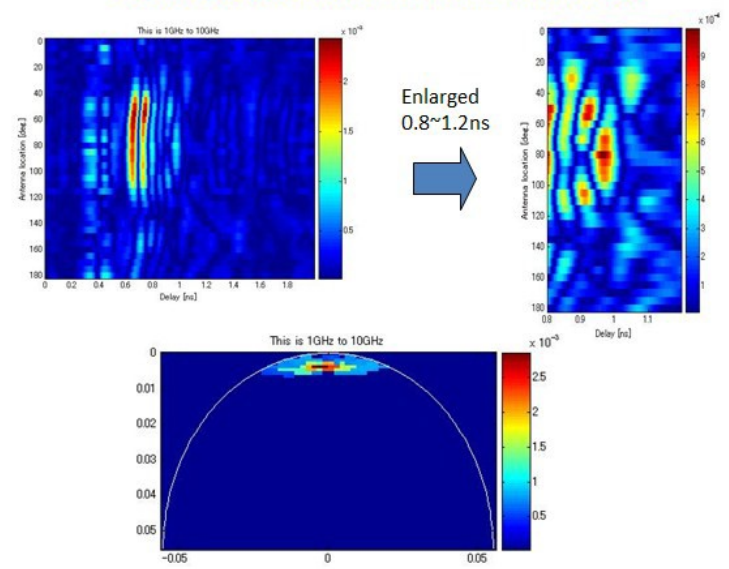

(a)

Object placed at $10 \mathrm{~mm}$ from the surface

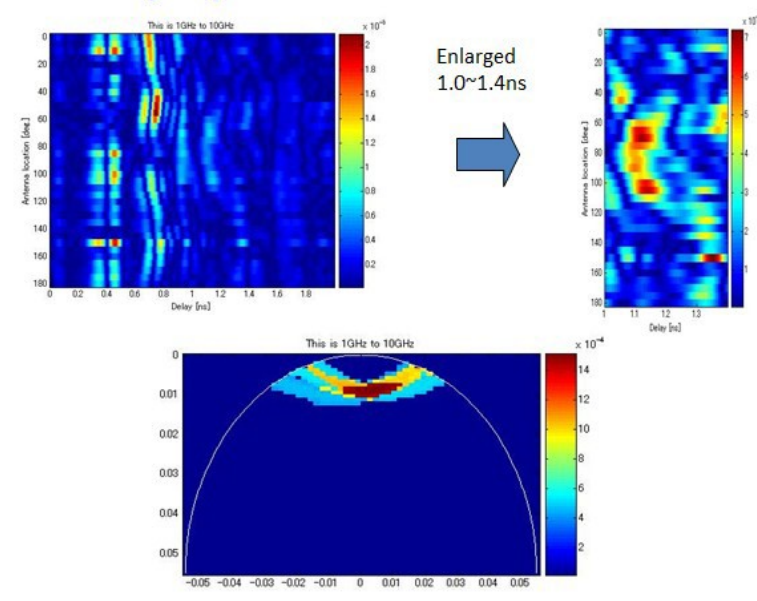

(b)

Object placed at $15 \mathrm{~mm}$ from the surface

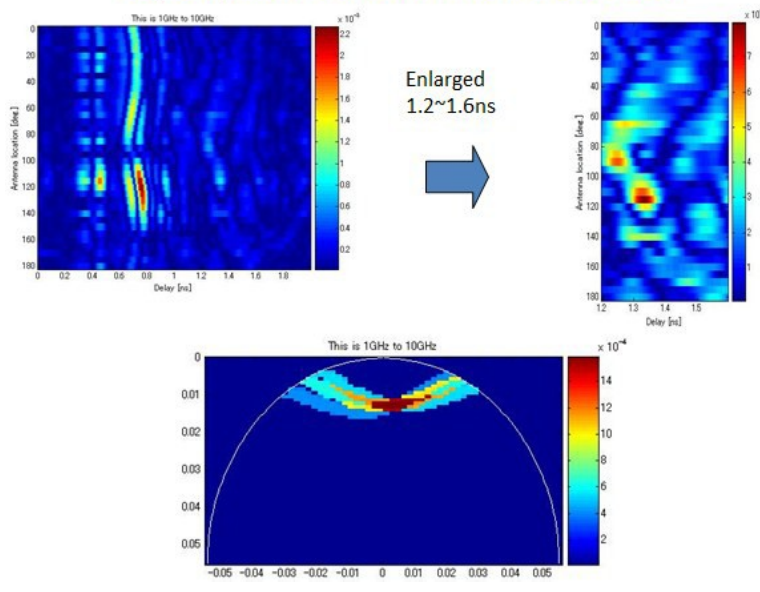

(c)

Fig. 4. The time delay and reconstructed permittivity profile for tumor placed at (a): $5 \mathrm{~mm}$; (b): $10 \mathrm{~mm}$ and (c): $15 \mathrm{~mm}$ from top of breast phantom. 
The data were preprocessed for background subtraction before using the focusing/image reconstruction algorithm. (Teflon covers are used for background subtraction and as shown in Fig 3(a-b)). The primary aim of this process is to equalize or time-aligned the scattered response from tumors as received by antennas in different locations.

The reconstructed results are shown in Fig. 4 for three different locations of tumor i.e. $5 \mathrm{~mm}, 10 \mathrm{~mm}$ and $15 \mathrm{~mm}$ from top surface of breast phantom. As can be seen the surface reflection levels are reduced by a factor of 4 compared to our previous measurements [10].

\section{ACKNOWLEDGEMENTS}

This work is partly supported by the Grant-in-Aid for Scientific Research, the Ministry of Education, Science, Sports and Culture (No. 20360186), and Grant for Practical Application of University R\&D Results under the Matching Fund Method, NEDO.

\section{References}

[1] J. J. Xia, T. M. Habashy, and J. A. Kong, "Profile inversion in a cylindrically stratified lossy medium,” Radio Sci., vol. 29, pp. 1131-1141, 1994.

[2] C. H. Jones, "Methods of breast imaging," Phys. Med. Biol., vol. 27, pp. 463-499, Apr. 1982.

[3] Y. J. Kim, L. Jofre, F. D. Flaviis, and M. Q. Feng, "Microwave reflection tomographic array for damage detection of civil structures," IEEE Trans. Microw. Theory Tech., vol. 51, no. 11, pp. 30223032, Nov. 2003.

[4] P. T. Huynh, A. M. Jarolimek, and S. Daye, “The false-negative mammogram,” Radiographics, vol. 18, pp. 1137-1154, 1998.

[5] Abubakar, P. M. van den Berg, and J. J. Mallorqui, "Imaging of biomedical data using a multiplicative regularized contrast source inversion method,” IEEE Trans. Microw. Theory Tech., vol. 59, no. 7, pp.1761-1771, Jul. 2002.

[6] A. Fhager, S. K. Padhi and J. Howard, "3D Image Reconstruction in Microwave Tomography Using an Efficient FDTD Model”, IEEE Antenna and Wireless Propagation Letters, vol: AWPL: 8, 2009, pp: 1353-1356.

[7] S. K. Padhi, A. Fhager, M. Persson and J. Howard, "Measured Antenna Response of a Proposed Microwave Tomography System Using an Efficient 3D-FDTD Model”, IEEE Antenna and Wireless Propagation Letters, vol: 7, 2008, pp: 689 - 692 .

[8] Dan Zhang, Atsushi Mase, Multi-objective experiment using ultrashort-pulse radar system for breast cancer detection', Proceeding of IRMMWTHz 2010, Rome, Sep. 2010.

[9] Takaichi S, Mase A et al., Simulation study and experiment of breast cancer using an ultrashortpulse radar, Proc 2008 Asia Pacific Microwave Conf (APMC2008), Macau Dec. 2008.

[10]Dan Zhang and A. Mase, "Radar-based breast cancer detection experiment with synthetic breast phantom using model subtraction method," Proceedings of APMC 2011, pp.207-210, Dec. 2011.

[11]E. C. Fear, X. Li, S. C. Hagness, and M. A. Stuchly, "Confocal microwave imaging for breast cancer detection: Localization of tumors in three dimensions," IEEE Trans. Biomed. Eng., vol. 49, no. 8, pp. 812-822, Aug. 2002.

[12] Hagness SC, Taflove A, Bridges JE, Twodimensional FDTD analysis of a pulsed microwave confocal system for breast cancer detection: Fixed-focus and antenna-array sensors, IEEE Trans Biomed Eng 45:1470 1479, 1998.

[13] Xu Li, Hagness SC, Confocal microwave imaging algorithm for breast cancer detection, IEEE Microw Wirel Compon Lett 11: 130 132, 2001.

[14] Bond EJ, Xu Li, Hagness SC, Microwave imaging via space-time beamforming for early detection of breast cancer, IEEE Trans Antennas Propag 51:1690 1705, 2003

[15] http://www.cirsinc.com/051 mri.html. 